\title{
HEAT TRANSFER COEFFICIENT IN STATIONARY HEATING-PLANE TYPE OF INDIRECT-HEAT AGITATED DRYER
}

\author{
TAKAO OHMORI, MORIO OKAZAKI AND RYOZO TOEI \\ Department of Chemical Engineering, Kyoto University, Kyoto 606
}

Key Words: Drying, Heat Transfer, Dryer, Heat Transfer Coefficient, Agitation, Granular Material

\begin{abstract}
The heat transfer coefficient between the heating wall and the granular bed in the stationary heating-plane type of indirect-heat agitated dryer was measured. Taking account of the effect of the clearance between heating wall and agitating blade, an improved heat transfer model was proposed. This model can also describe the dependence of the heat transfer coefficient on the circumferential velocity of the agitator, the thermal properties of the granular material, the particle size and so on. Furthermore, it was confirmed that this model was usable when the particles were non-spherical or when scale-up of the dryer was considered.
\end{abstract}

\section{Introduction}

The indirect-heat agitated dryer has been widely used recently in many fields because of its high thermal efficiency and minimal exhaust gas. This work is concerned with the heat transfer in such dryers of the stationary heating-plane type. ${ }^{6)}$ In this type, there inevitably exists a clearance between heating wall and agitating blade, and the negative effect of this clearance on heat transfer is very significant.

It also becomes important to estimate quantitatively the effect of clearance on the heat transfer coefficient when scale-up of the dryer is considered. The width of the clearance cannot be kept quite small, since in that case the agitator shaft might bend in a large-scale dryer. In that case, the effect of the dryer diameter on the heat transfer coefficient is also a problem to be investigated.

The granular material particles to be processed in this dryer are non-spherical in many cases. Therefore, it is also important to investigate the effect of particle shape on the heat transfer coefficient.

\section{Model}

In the previous model proposed by the present authors, ${ }^{6}$ ) heat transfer in the stationary heatingplane type of indirect-heat agitated dryer consists of the four mechanisms shown in Fig. 1.

1) wall-to-particle heat transfer

2) heat conduction in packed bed

3) heat convection by particle motion in bulk material

Received July 6, 1985. Correspondence concerning this article should be addressed to M. Okazaki. T. Ohmori is now at National Chemical Laboratory for Industry, Tsukuba, Ibaraki 305 . R. Toei is now at Toyama National College of Technology, Toyama 939.
4) heat transfer in clearance

Based on the model suggested by Schlünder ${ }^{3-5)}$ and partly revised by Mollekopf and Martin, ${ }^{2)}$ the wall-to-particle heat transfer coefficient can be calculated with the following equations if the particle is spherical:

$$
\begin{gathered}
h_{p}=4\left(\lambda_{g} / d_{p}\right)\left\{\left(1+2 \sigma / d_{p}\right) \ln \left(1+d_{p} / 2 \sigma\right)-1\right\} \\
\sigma=2 \frac{2-\gamma}{\gamma} \sqrt{2 \pi R T / M} \frac{\lambda_{g}}{p\left(2 c_{p g}-R / M\right)} \\
h_{s}=\psi h_{p}+(1-\psi) h_{2 p}+h_{R}
\end{gathered}
$$

In these equations, $h_{p}$ is the maximum achievable wall-to-single particle heat transfer coefficient, $h_{s}$ is the maximum achievable wall-to-first particle layer coefficient, $h_{2 p}$ is the coefficient between the heating plane and a second-layer particle from the plane and $h_{R}$ is that by radiation. The second and third terms in Eq. (3) are negligible in comparison with the first term so long as the pressure and temperature are atmospheric.

The heat transfer coefficient of heat conduction in the packed bed is obtained by the penetration model, in which the packed bed is regarded as

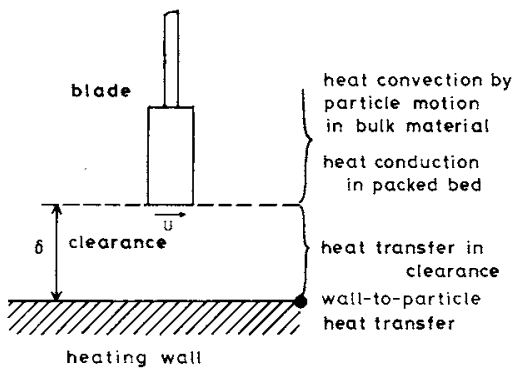

Fig. 1. Heat transfer mechanism. 
homogeneous. ${ }^{1)}$

$$
h_{c}=\sqrt{\lambda_{e} c_{p m} \rho_{b} / \pi t}
$$

The heat convection by particle motion has not yet been analysed quantitatively because the particle motion is not well known, as Schlünder mentioned. ${ }^{5)}$ However, its effect on heat transfer can be neglected if bulk material particles are mixed perfectly when the agitating blade scrapes them. It should be noted that the present model is only applicable under this condition.

As there is a clearance between the heating wall and the agitating blade, the heat transfer resistance in this part must also be taken into account. There may be some velocity distribution of particles in this clearance, but it is difficult to estimate. We therefore introduce the hypothesis that there exists a stationary particle layer, which has an effective thickness $\delta_{e}$, on the heating wall. According to this hypothesis, the heat transfer resistance in this part is equal to $\delta_{e} / \lambda_{e}$.

When the resistances mentioned above are connected in series to each other, we can get the instantaneous heat transfer coefficient as follows:

$$
h_{i}=1 /\left(1 / h_{s}+\delta_{e} / \lambda_{e}+1 / h_{c}\right)
$$

Furthermore, it is assumed that the contact time between particle and heating wall is equal to the interval of scraping by the agitating blade. So the time-averaged heat transfer coefficient can be obtained by integrating Eq. (5) during this contact time.

$$
\begin{gathered}
h_{w}=\left(\int_{0}^{\tau} h_{i} d t\right) / \tau \\
=2 h_{s} \lambda_{e}\left\{\sqrt{\pi \tau^{\circ}}-\ln \left(1+\sqrt{\pi \tau^{\circ}}\right)\right\} /\left\{\left(\lambda_{e}+\delta_{e} h_{s}\right) \pi \tau^{\circ}\right\} \\
\tau^{\circ}=h_{s}^{2} \lambda_{e} \tau /\left\{\left(\lambda_{e}+\delta_{e} h_{s}\right)^{2} c_{p m} \rho_{b}\right\}
\end{gathered}
$$

The relation between the contact time and the circumferential velocity of the agitator depends on the agitator's structure. When the agitating blade scrapes a given point of wall once per revolution, the relation may be described by Eq. (8). Equation (9) shows the relation when the agitating blade scrapes a given point twice per revolution at regular intervals.

$$
\begin{aligned}
& \tau=\pi\left(D_{d}-2 \delta\right) / U \\
& \tau=\pi\left(D_{d}-2 \delta\right) / 2 U
\end{aligned}
$$

Finally, we can calculate the heat transfer coefficient by Eqs. (1)-(3), (6), (7) and (8) or (9) if $\delta_{e}$ is known.

Our previous model had two problems in the matter of estimating $\delta_{e^{\cdot}}{ }^{6)}$ One was the assumption that the heat transfer resistance in the clearance did not change even if the circumferential velocity of the agitator changed. The other was that the previous model included parameters that depend on the type of agitator. To solve these problems, the following improved model is proposed here.

The effective thickness $\delta_{e}$ is thought to depend mainly on the width of the clearance $\delta$, the particle diameter $d_{p}$, the velocity of the agitating blade $U$ and the relative velocity between the particles and the agitating blade along the lateral plane of the blade $U_{B}$ (Fig. 2). $U_{B}$ was introduced in order to consider the effect of the agitator's structure on heat transfer in the clearance. So we postulate the following form, considering the extreme cases.

$$
\begin{array}{ll}
\delta / d_{p} \geqq 1: & \delta_{e} / d_{p}=1 /\left(1 / \xi+d_{p} / \delta\right) \\
& \xi=a\left(\delta / d_{p}-1\right)^{b} /\left(U^{c}+d U_{B}{ }^{e}\right) \\
& U_{B}=U \sin \beta \\
0 \leqq \delta / d_{p} \leqq 1: & \delta_{e}=0
\end{array}
$$

where $a, b, c, d$ and $e$ are constants which should be determined by fitting experimental data. The dimensionless variable $\xi$ is an index indicating the variation of effective thickness. It should be noted that the dimensions of $U$ and $U_{B}$ are meters per second in Eq. (11). In Eqs. (10)-(12), $\delta_{e}$ reaches zero if $U$ becomes infinite and $\delta_{e}$ reaches $\delta$ if $U$ becomes zero.

\section{Experimental}

Two sizes of dryers, with diameters $D_{d}$ of $20 \mathrm{~cm}$ and $60 \mathrm{~cm}$, were used for the experiments. The smaller one was the same as used in the previous work. ${ }^{6}$ A schematic diagram of the experimental apparatus using the larger dryer is shown in Fig. 3.

The temperature of the wall was kept constant by adjusting the steam flow rate. To avoid heat transfer from the granular bed to the end-plates of the dryer and to the air over the bed surface, hot water was circulated in the jackets installed in the end-plates and hot air was blown over the bed surface. Heaters for both water and air were controlled to keep their temperatures equal to that of the granular bed.

Two types of agitators, the double-spiral type $(\beta=$ $\left.65^{\circ}, \delta=0.7-5.6 \mathrm{~mm}\right)$ and the flat-bar type $\left(\beta=0^{\circ}, \delta=\right.$ $0.75-10.1 \mathrm{~mm}$ ), were used in the experiments. Figures 4 and 5 show them used in the smaller dryer. These agitators scraped a given point of the wall once per revolution. The agitator used in the larger dryer was almost the same double-spiral type. In this agitator, however, $\beta=80^{\circ}, \delta=0.65 \mathrm{~mm}$ and a given point was scraped twice per revolution at regular intervals.

The procedure of the experiments was almost the same as in the previous work. ${ }^{6)}$ The heat transfer coefficient was calculated from the following equation by use of data obtained:

$$
h_{w}=\left(W_{m} c_{p m} \frac{d T_{b}}{d t}+W_{s h} c_{p s h} \frac{d T_{s h}}{d t}\right) /\left\{A_{w}\left(T_{w}-T_{b}\right)\right\}
$$


Table 1. Granular materials

\begin{tabular}{llcccc}
\hline Material & Shape & $\begin{array}{c}d_{p} \\
{[\mathrm{~mm}]}\end{array}$ & $\begin{array}{c}\rho_{b} \\
{\left[\mathrm{~kg} \cdot \mathrm{m}^{-3}\right]}\end{array}$ & $\begin{array}{c}c_{p m} \\
{\left[\mathrm{~J} \cdot \mathrm{kg}^{-1} \cdot \mathrm{K}^{-1}\right]}\end{array}$ & $\begin{array}{c}\lambda_{e} \\
{\left[\mathrm{~W} \cdot \mathrm{m}^{-1} \cdot \mathrm{K}^{-1}\right]}\end{array}$ \\
\hline Glass beads A & Sphere & 0.36 & 1450 & 853 & 0.203 \\
Glass beads B & Sphere & 1.1 & 1450 & 853 & 0.203 \\
Activated alumina & Sphere & 0.51 & 910 & 920 & 0.157 \\
Acrylic resin & Sphere & 0.57 & 690 & 1560 & 0.116 \\
Millet & Flat oval & 1.7 & 820 & $2300^{*}$ & 0.170 \\
\hline
\end{tabular}

* Measured under the condition of complete drying.

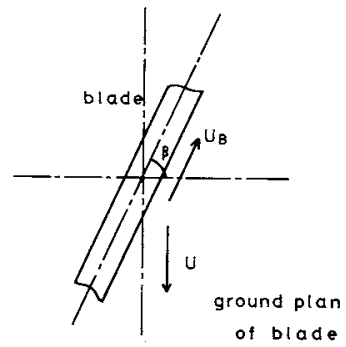

Fig. 2. Relative velocity between agitating blade and particles along lateral plane of blade.

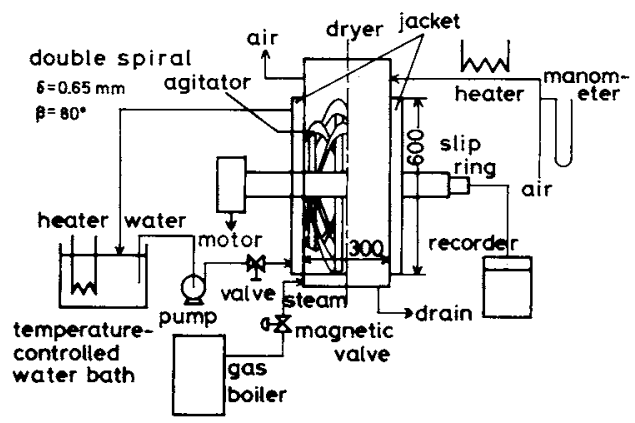

Fig. 3. Schematic diagram of experimental apparatus with large dryer.

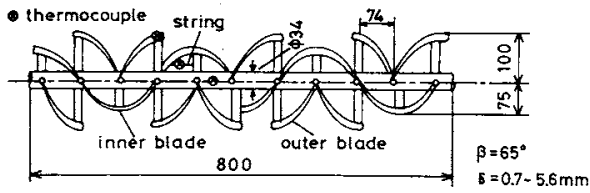

Fig. 4. Double-spiral agitator.

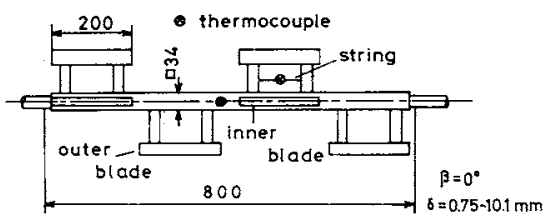

Fig. 5. Flat-bar agitator.

Equation (14) can be obtained by the heat balance in the system of both the granular bed and the agitator. The holdup of the granular material was about $55 \%$ in the small dryer, and about $35 \%$ in the large dryer.

Five kinds of granular materials were used in the experiments. Their properties are tabulated in Table
1. The effective thermal conductivities of the granular beds shown in this table were measured by the Q.T.M. rapid thermal conductivity meter (manufactured by Showa Denko Co., Ltd.; QTM-D1) and the apparent densities of the beds and the specific heat at constant pressure of the millet were also obtained by our measurements.

\section{Results and Discussion}

The experimental heat transfer coefficients are plotted as a function of the circumferential velocity of the double-spiral agitator in Figs. $6-8$ and as a function of that of the flat-bar agitator in Figs. 9 and 10. These results were obtained in the smaller dryer. As a result of determining the parameters in Eq. (11), which provided the best agreement between data points and the curves calculated from the present model, $a=0.6$, $b=0.5, c=0.8, d=3.5$ and $e=0.45$ were obtained. The curves calculated from the model with use of these values are also shown in Figs. 6-10. Agreement between the observed data and these curves seems satisfactory.

To illustrate the effect of the clearance on the heat transfer coefficient, the observed $h_{w}$ values for $U=$ $0.21 \mathrm{~m} \cdot \mathrm{s}^{-1}$ and $U=0.78 \mathrm{~m} \cdot \mathrm{s}^{-1}$ are plotted as a function of the dimensionless clearance $\delta / d_{p}$ in Figs. 11 and 12. As shown in these figures, the calculated heat transfer coefficient is constant when $\delta / d_{p}$ is less than unity. Then the calculated $h_{w}$ decreases with increasing $\delta / d_{p}$ if $\delta / d_{p}$ is greater than unity. The manner of its decrease has the following characteristics:

1) The value of $h_{w}$ decreases rapidly if $\delta / d_{p}$ is over unity.

2) The value of $h_{w}$ decreases moderately in the region of $\delta / d_{p}=2-10$.

3) The gradient of decrease becomes large again if $\delta / d_{p}$ is more than 10 .

It is also shown in those figures that $\delta / d_{p}$ has a greater influence on $h_{w}$ with the flat-bar agitator than with the double-spiral type. For different particle sizes and circumferential velocities of the agitator, the influence of $\delta / d_{p}$ and the effect of the type of agitator on $h_{w}$ are almost the same, though the absolute values of $h_{w}$ have certain differences. 


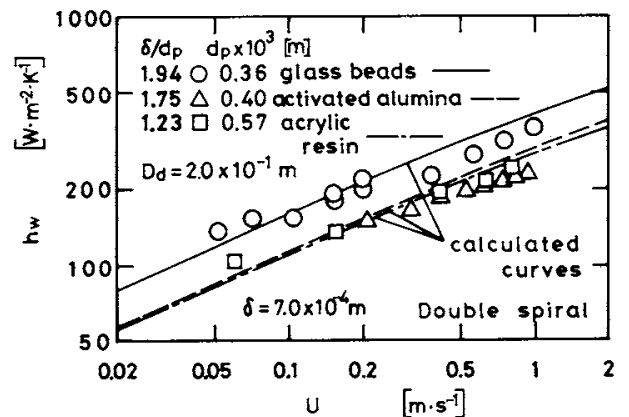

Fig. 6. Heat transfer coefficient vs. circumferential velocity of double-spiral agitator.

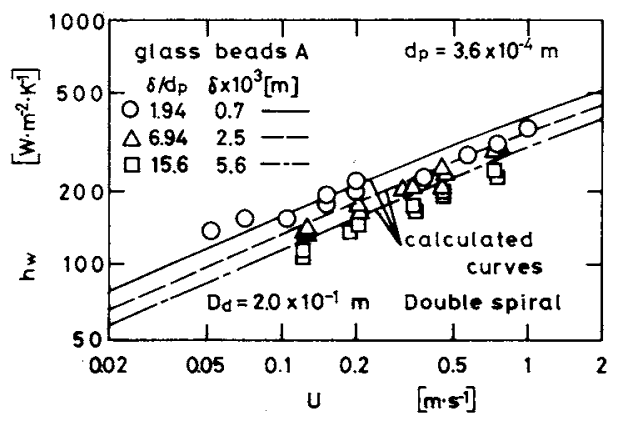

Fig. 7. Heat transfer coefficient vs. circumferential velocity of double-spiral agitator.

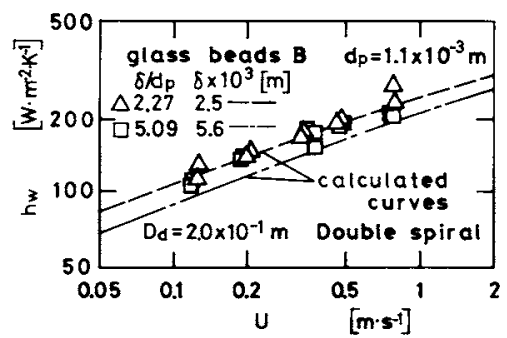

Fig. 8. Heat transfer coefficient vs. circumferential velocity of double-spiral agitator.

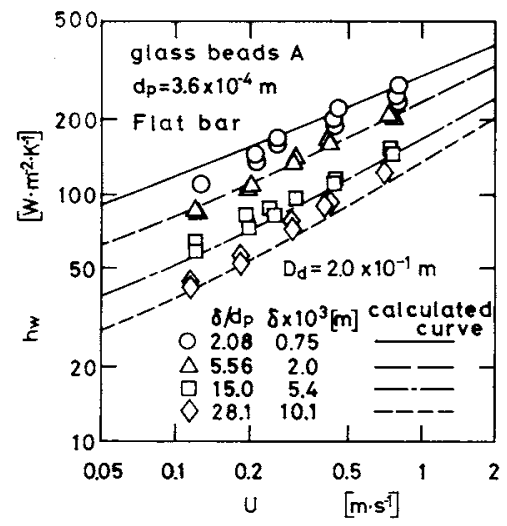

Fig. 9. Heat transfer coefficient vs, circumferential velocity of flat-bar agitator.

In Fig. 13, calculated $h_{w}$ vs. the experimental $h_{w}$ values are shown for all data. Almost all data are correlated within $\pm 25 \%$. So it is concluded that the agreement between calculated and experimental $h_{w}$ is

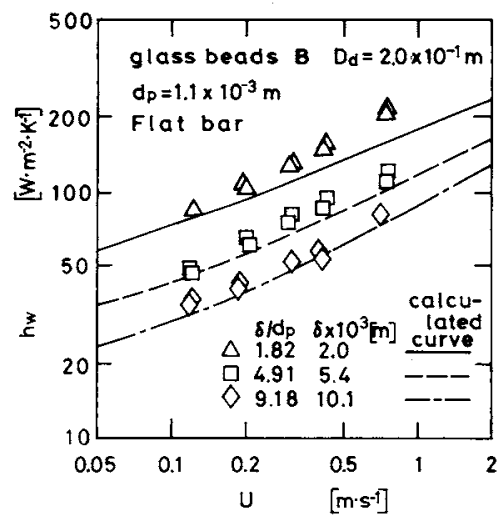

Fig. 10. Heat transfer coefficient vs. circumferential velocity of flat-bar agitator.

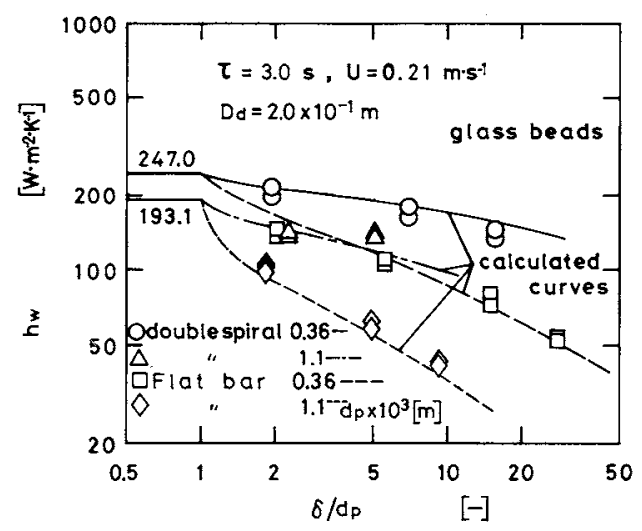

Fig. 11. Heat transfer coefficient vs. dimensionless clearance.

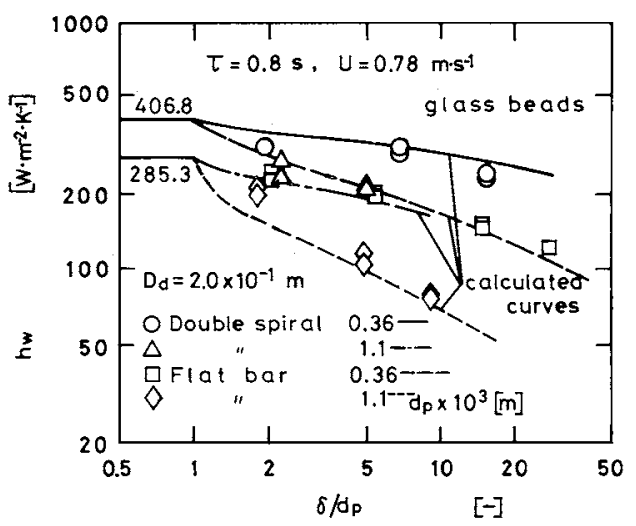

Fig. 12. Heat transfer coefficient vs. dimensionless clearance.

good under the condition that the particle shape is spherical.

Figure 14 shows the relation of $h_{w}$ vs. $U$ of the double-spiral agitator for the millet grain, which is a non-spherical particle. As shown, there is a serious deviation between the experimental heat transfer coefficient and the calculated curves when the average particle diameter $d_{p}=1.7 \mathrm{~mm}$, obtained by sieving, was used in the calculation provided that the particle shape was spherical. The wall-to-single particle heat transfer coefficient naturally depends on particle 


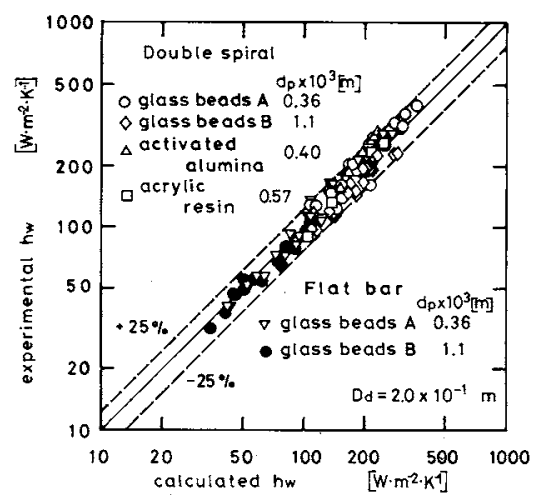

Fig. 13. Experimental $h_{w}$ vs. calculated $h_{w}$.

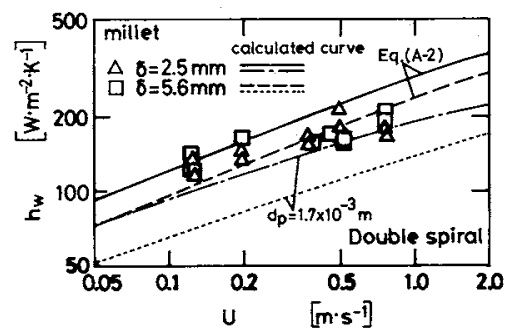

Fig. 14. Heat transfer coefficient vs. circumferential velocity of double-spiral agitator.

shape. ${ }^{2,3)}$ So it is necessary to determine the shape of the millet particles quantitatively, details of which are given in the Appendix.

The calculated curves obtained in that way are also presented in Fig. 14, and their comparison with the experimental data showed no serious deviation. So it might be concluded that the heat transfer coefficient where the particle shape is non-spherical can be predicted with the present model.

To investigate the effect of the dryer diameter on the heat transfer coefficient, the experimental heat transfer coefficients for the large dryer are plotted as a function of the double-spiral agitator in Fig. 15. The curve presented in this figure is that obtained from Eqs. (1)-(3), (6), (7) and (9)-(12) using the same parameters as obtained for the smaller dryer. Agreement between observed and calculated heat transfer coefficients is fairly good. It is therefore concluded that the present model is also applicable in cases where scale-up of the dryer is considered.

\section{Conclusion}

The coefficient of heat transfer between the heating wall and the granular bed in the stationary heatingplane type of indirect-heat agitated dryer was measured. An improved heat transfer model, considering the effect of the clearance between the heating wall and the agitating blade, was proposed. The comparison between experimental and the calculated heat transfer coefficients showed fairly good agreement. Furthermore, it was confirmed that this model is

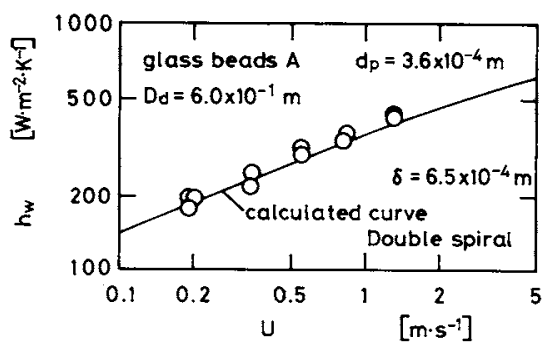

Fig. 15. Heat transfer coefficient vs. circumferential velocity of double-spiral agitator with large dryer.

usable where the particle shape is non-spherical or where scale-up of the dryer is considered.

\section{Appendix}

The local wall-to-single particle heat transfer coefficient at a given location is given by Eq. $(\mathrm{A}-1){ }^{2-5)}$

$$
h_{p, l}=\lambda_{g} /(s+\sigma)
$$

where $s$ is the local gap width between the heating wall and the particle surface (Fig. A-1) and $\sigma$ is the modified mean free path of interstitial gas molecule defined by Eq. (2). The integration of Eq. (A-1) gives the average value $h_{p}$ with respect to the projection area of the particle. If the particle shape is spherical, this integration can be done analytically and gives Eq. (1). In the case of non-spherical particles, it is necessary to determine the particle shape quantitatively in order to estimate the value of $s$. Then we make the following assumptions (Fig. A-1).

1) The cross section of millet grains is described by Eq. (A-2).

2) The millet grain is rotation-symmetric against the $y$-axis. The direction of contact between the particle and the wall was determined by our observation.

$$
(x / \varepsilon)^{n}+(y / \kappa)^{n}=1, \quad n=2.2
$$

With these assumptions, the value of $s$ can be obtained and the average value $h_{p}$ in this case can be calculated numerically.

\section{Acknowledgement}

The authors are grateful to Messrs. M. Miyahara, S. Araki and $\mathrm{H}$. Ohmae for their assistance in this work and to Kurimoto Ltd. for assembling the experimental apparatus.

\section{Nomenclature}

\section{(a)}

$$
d
$$

$$
\text { a }
$$$$
\text { h }
$$

$=$ heat transfer area

$=$ constant in Eq. (11)

$=$ constant in Eq. (11)

$=$ constant in Eq. (11)

$=$ specific heat at constant pressure $\left[\mathrm{J} \cdot \mathrm{kg}^{-1} \cdot \mathrm{K}^{-1}\right]$

$c_{p}$

$=$ diameter of dryer

$=$ constant in Eq. (11)

$=$ particle diameter

$=$ constant in Eq. (11)

$=$ heat transfer coefficient

$=$ time-averaged heat transfer coefficient between heating wall and granular bed

$=$ molar weight of interstitial gas molecule

$$
\left[\mathrm{W} \cdot \mathrm{m}^{-2} \cdot \mathrm{K}^{-1}\right]
$$

$\left[\mathrm{kg} \cdot \mathrm{kmol}^{-1}\right]$

$=$ parameter in Eq. $(\mathrm{A}-2)$

[-]

$=$ pressure

[Pa]

$=$ gas constant $\quad\left[\mathrm{J} \cdot \mathrm{kmol}^{-1} \cdot \mathrm{K}^{-1}\right]$

$=$ local gap width between heating wall and particle surface (Fig. A-1) 


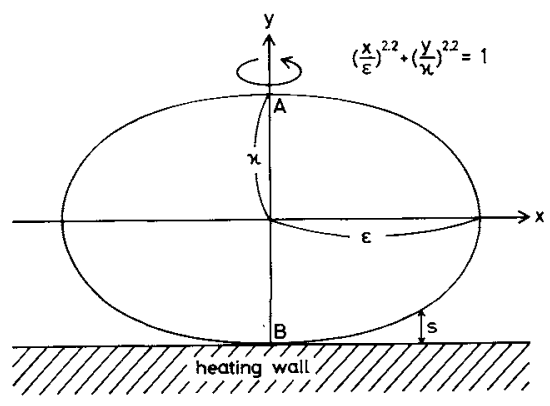

Fig. A-1. Cross section of millet.

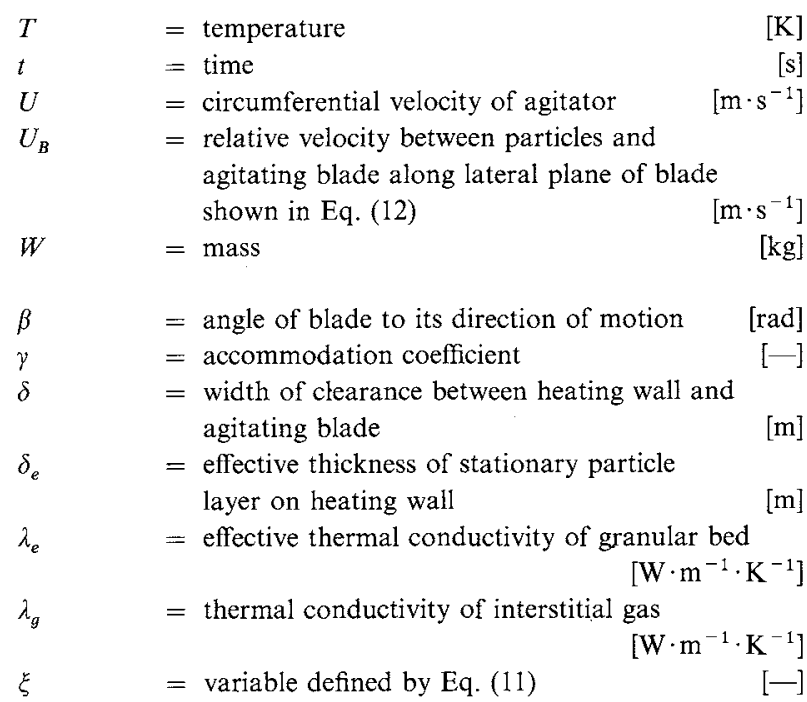

$\rho_{b} \quad=$ apparent density of granular bed $\quad\left[\mathrm{kg} \cdot \mathrm{m}^{-3}\right]$
$=$ modified contact time defined by Eq. (7)

$=$ surface coverage factor

\section{〈Subscripts〉}

$$
\begin{array}{ll}
b & =\text { granular bed } \\
c & =\text { conduction } \\
g & =\text { interstitial gas } \\
i & =\text { instantaneous value } \\
l & =\text { local value } \\
m & \doteq \text { granular material } \\
p & =\text { particle } \\
R & =\text { radiation } \\
s & =\text { particle layer } \\
s h & =\text { shaft of agitator } \\
w & =\text { wall }
\end{array}
$$

\section{Literature Cited}

1) Krischer, O. and W. "Kast: "Die wissenschaftlichen Grundlagen der Trocknungstechnik," 3rd ed., SpringerVerlag, Berlin (1978).

2) Mollekopf, N. and H. Martin: Verfahrenstechnik, 16, 701 (1982).

3) Schlünder, E. U.: Chem.-Ing.-Tech., 43, 651 (1971) [Int. Chem. Eng., 20, 550 (1980)].

4) Schïnder, E. U.: Chem.-Ing.-Tech., 53, 925 (1981).

5) Schï̈nder, E. U.: Proc. 7th Int. Heat Transfer Conf., Munich, Vol. 1, p. 195 (1982).

6) Toei, R., T. Ohmori, T. Furuta and M. Okazaki: Chem. Eng. Process., 18, 149 (1984). 